\title{
Experimental Realization of a Rotational Ratchet in a Granular Gas
}

\author{
Peter Eshuis, ${ }^{1}$ Ko van der Weele, ${ }^{2}$ Detlef Lohse, ${ }^{1}$ and Devaraj van der Meer ${ }^{1}$ \\ ${ }^{1}$ Physics of Fluids, University of Twente, Post Office Box 217, 7500 AE Enschede, The Netherlands \\ ${ }^{2}$ Mathematics Department, University of Patras, 26500 Patras, Greece \\ (Received 10 March 2009; revised manuscript received 29 October 2009; published 16 June 2010)
}

\begin{abstract}
We construct a ratchet of the Smoluchowski-Feynman type, consisting of four vanes that are allowed to rotate freely in a vibrofluidized granular gas. The necessary out-of-equilibrium environment is provided by the inelastically colliding grains, and the equally crucial symmetry breaking by applying a soft coating to one side of each vane. The onset of the ratchet effect occurs at a critical shaking strength via a smooth, continuous phase transition. For very strong shaking the vanes interact actively with the gas and a convection roll develops, sustaining the rotation of the vanes.
\end{abstract}

Introduction.-Throughout the ages scientists and laymen alike have tried to find a way to circumvent the second law of thermodynamics and create work out of thermal noise. In 1912, Marian Smoluchowski devised an especially appealing thought experiment [1], which consisted of four vanes and an asymmetrically toothed wheel with a pawl, submerged in a molecular heat bath [Fig. 1(a)]. At first glance it seems that the wheel can turn in one direction only, in violation of the second law. However, Richard Feynman unambiguously showed that this type of device does not produce work at thermal equilibrium [2]: Since not only the vanes but also the pawl are subject to collisions with the gas molecules, the pawl bounces off the toothed wheel and causes the system to rotate randomly in either direction.

In contrast, systems outside of thermal equilibrium are very well capable of creating work (directed motion) out of a noisy environment by means of the ratchet effect [3]. In fact, ratchet type devices have been proposed as the paradigmatic way in which motors operate at Brownian scales [4], and during the last decade scientists have realized (over a limited rotation range) a molecular version of Smoluchowski's device [5]. Here-on a macroscopic level-we construct a fully operational rotational ratchet of the Smoluchowski-Feynman type, capable of any uninterrupted number of revolutions. It consists of four vanes that are allowed to rotate freely in a granular gas. The necessary out-of-equilibrium environment-to bypass the second law of thermodynamics which prohibits the extraction of work from a system at thermal equilibrium-is provided by the granular gas. This is by its very nature out of equilibrium since in order to sustain the gaseous state it requires an external energy input to balance the energy dissipation caused by the inelastically colliding particles. The equally essential second ingredient, the symmetry breaking that must be present in order to rectify the stochastic motion due to the noisy environment, is provided by the fact that the two sides of each vane are coated differently. Although similar devices have been considered in a granular gas from a theoretical point of view [6,7], we here present the first experimental realization.

Our setup is sketched in Fig. 1(b). The four vanes $(25 \times$ $60 \mathrm{~mm}^{2}$ each, made from one piece of stainless steel) are precisely balanced around the axis, which is connected to the container wall by a low-friction ball bearing. The angle $\theta(t)$ is measured (at $1000 \mathrm{~Hz}$ ) by an optical angle encoder, with an accuracy of $1.9 \times 10^{-7} \mathrm{rad}$. Thanks to the small moment of inertia of the vanes and sensor $(I=7.2 \times$ $10^{-5} \mathrm{~kg} \mathrm{~m}^{2}$ ) single collisions of the particles with the vanes are easily detected. The granular particles in our setup are glass beads of diameter $d=4.0 \mathrm{~mm}(\rho=$ $2.6 \mathrm{~g} / \mathrm{cm}^{3}$ ). They are brought into a gaslike state by the vibrating bottom, which is mounted on a shaker with tunable frequency $f$ and amplitude $a$. The container is not connected to this bottom: It is a stationary perspex

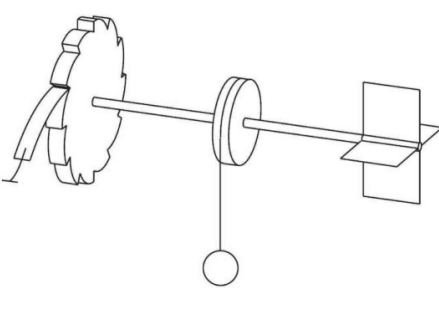

(a)



(b)
FIG. 1. (a) The gedanken experiment of Smoluchowski [1] as envisaged by Feynman [2]. (Our movie of the original design operating in a granular gas has been attached as EPAPS_2 [8].) (b) Our granular realization of the Smoluchowski-Feynman ratchet. The vanes can rotate freely in both directions when hit by the granular particles, and their angle $\theta(t)$ is recorded by the rotation sensor. The asymmetric coating of the vanes induces a preferential direction of rotation: the ratchet effect. The height $h$ (between the null position of the vibrating bottom and the vanes) can be varied. 
cage $\left(140 \times 140 \times 400 \mathrm{~mm}^{3}\right)$ with a meshed top in order to prevent particles from leaving the system and at the same time keeping the air pressure constant. The collisions with the particles cause a stochastic motion of the vanes around the axis.

The natural dimensionless control parameters for this system are: (i) The number of particles $N$ (500, 1000, and 2000 in the experiments presented here); (ii) The dimensionless height $h / d$ of the vanes above the vibrating bottom (in our experiments $h=51$ or $75 \mathrm{~mm}$ ); (iii) the coefficient of normal restitution $e$ of the particle-particle collisions (we use glass spheres with $e \approx 0.95$ ); and (iv) the shaking strength $S=4 \pi^{2}(a f)^{2} /(g h)$, which is the ratio of the typical kinetic energy given to the particles by the vibrating bottom and their potential energy at the height of the vanes. The shaking strength $S$ is the parameter which we will vary systematically.

Symmetrically coated vanes.-We first study the symmetric version of the system, in which the two sides of each vane are identical. Provided the setup is carefully balanced, there is no preferential direction for the rotation in this case, i.e., no ratchet effect. Figure 2 shows the results at four increasing values of the shaking strength $S$ :

(a) At mild shaking, the bulk of the granular gas remains close to the bottom and only occasionally does a particle jump high enough to hit the vanes. These will be moved into an $\times$ position, as sketched, which is stabilized through the following mechanism: The most energetic particles come straight from the bottom, having a velocity mainly in the vertical direction. Upon hitting the vanes in the $X$ position, they are reflected towards the neighboring lower vane; the ensuing collision with this vane redirects them back to the bottom. These two collisions have an opposite effect on the rotation of the vanes and therefore neutralize each other. This stabilizing mechanism is absent for the + position, where the particles are more likely to collide only once with the vanes. We find a stable $X$ position for all studied values of the particle number $N$, which suggests that the state is present at all densities (although small particle numbers tend to increase the escape rate from the $X$ position). Since all available theories are based on uncorrelated collisions, the existence of this stable position was not predicted $[6,7]$.

When the shaking strength is increased, the two top vanes in the $\times$ configuration start to act as a particle trap. The system periodically unloads the trapped particles, and then reassumes an $\times$ configuration. For growing $S$ the system has to unload more frequently, because the center of mass of the granular gas moves closer towards the vanes and hence more particles are caught per unit time.

(b) Above a critical value $S_{\mathrm{cr}}$, the rocking motion due to these repeated discharges turns into an unbiased random walk between the four equivalent $\times$ positions [Fig. 2(b)]. That is, the vanes now explore a widening range of angles following the diffusion law $\left\langle\theta^{2}(t)\right\rangle=2 D t$, where the dif-
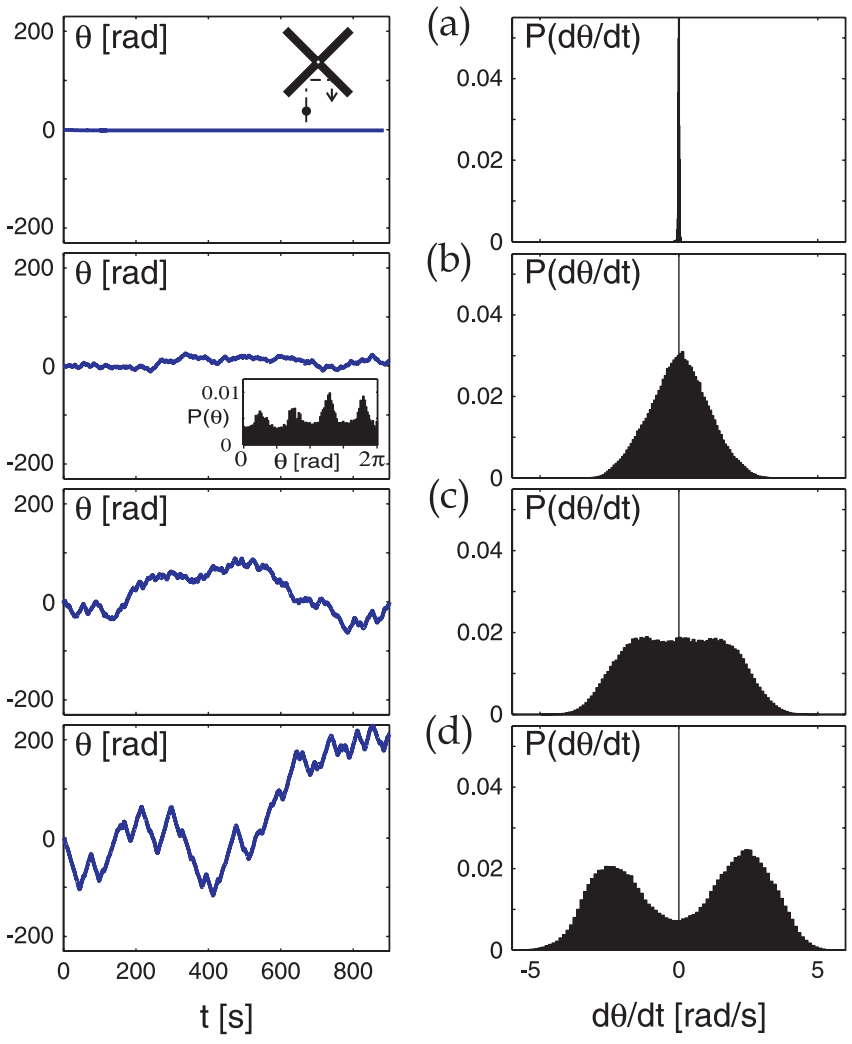

FIG. 2 (color online). Symmetrically coated vanes. The angle $\theta(t)$ and the angular velocity distribution $P(d \theta / d t)$ for $N=$ 2000 particles and $h=51 \mathrm{~mm}$ at four different shaking strengths: (a) amplitude $a=1.5 \mathrm{~mm}$, frequency $f=50 \mathrm{~Hz}$ [i.e., $S=0.44$ ]; (b) $a=1.5 \mathrm{~mm}, f=110 \mathrm{~Hz}[S=2.15$ ], the inset gives the distribution of $\theta(t)(\bmod 2 \pi)$ showing a marked preference for the $\times$ position; (c) $a=3.5 \mathrm{~mm}, f=55 \mathrm{~Hz}[S=$ 2.92]; (d) $a=3.5 \mathrm{~mm}, f=65 \mathrm{~Hz}[S=4.08]$.

fusion coefficient $D$ grows with increasing shaking strength. Here, the distribution of the angular velocities is consistent with a Gaussian, with an increased probability for zero velocity which can be traced back to stick-slip motion in the ball bearings. The distribution of the angle $\theta(t)(\bmod 2 \pi)$ over time still shows a preference for the $X$ positions (see inset); this feature diminishes gradually with increasing $S$ until at very strong shaking the distribution becomes uniform.

(c) In Fig. 2(c) we encounter a second transition: The probability distribution function of the angular velocity $d \theta / d t$ develops a double maximum. The system acquires a preference for a specific value of the angular velocity, with equal likelihood regarding the clockwise and counterclockwise direction. This is due to the fact that the rotating vanes induce a convective motion of the granular gas in the same direction. To obtain accurate information on density, velocity, and granular temperature of the gas we performed three-dimensional molecular dynamics (MD) simulations, in which the grains are modeled as frictionless hard spheres with a normal restitution coefficient $e=0.90$, which also 
interact inelastically with the vanes. While the vanes rotate in one direction only, we observe a clear convection roll in the granular gas [see Fig. 4(a)]. In addition, the simulations show that the particles trapped between the two upper vanes, when toppling over to one side, make the granular gas at that side denser and thus less energetic. Therefore the kicks from the particles at the other side are more powerful, with the result that the rotational direction initialized by the toppling is now reinforced. Only a sufficiently large statistical fluctuation will reverse the direction, and then the same argument applies to the opposite rotation mode. Additional information on the simulation results, including a movie, clearly shows how the convection roll is connected to density and (granular) temperature gradients in the gas [8].

(d) At vigorous shaking, Fig. 2(d), the double maximum is evident: The angular velocity is either strongly positive or negative, but rarely close to zero. This yields a zigzag pattern in the plot of $\theta$ versus $t$. If the shaking strength is increased still further, the double maximum gradually disappears again; the particles become simply too energetic to get trapped between the vanes anymore, thus disabling the mechanism described above.

The symmetry breaking discussed in (c) and (d) is a prime example of a system in which the heat bath itself is affected by the presence of the device. The formation of the convection roll depends on the particle number (for $N=$ 500 it is absent) and the orientation of the vanes: The symmetry breaking is not observed when in the simulation the axis is positioned perpendicular to the vibrating bottom. The result is robust: If in our simulations we extend the lateral size of the system (while keeping the number of particles per unit area of the bottom constant) a convection roll still forms around the vane area, with smaller counterrotating rolls in the corners.

Asymmetrically coated vanes: Ratchet.-In order to turn the system into a ratchet, we now introduce a slight asymmetry. We do this by changing the symmetry of the vanes themselves - instead of introducing the ratchet and pawl of Fig. 1(a) - to avoid spatial granular temperature fluctuations in the gas [8]. The left hand side of each vane is coated with rubber tape, which makes this side considerably softer, diminishing its coefficient of normal restitution. So at this side the kinetic energy from the colliding particles is dissipated more than on the other side, or stated differently, the collisions at the soft side are less effective in moving the vane. As a result the vanes are expected to preferentially rotate in the counter-clockwise (positive $\theta$ ) direction. This is explored in Fig. 3, which is the asymmetric counterpart of Fig. 2(b)-2(d).

At very low shaking strengths, when there is hardly any interaction yet with the granular gas, the vanes flutter around the $X$ position just as in the symmetric case and the angular velocity distribution is indistinguishable from the symmetric case (not shown). It takes a certain threshold
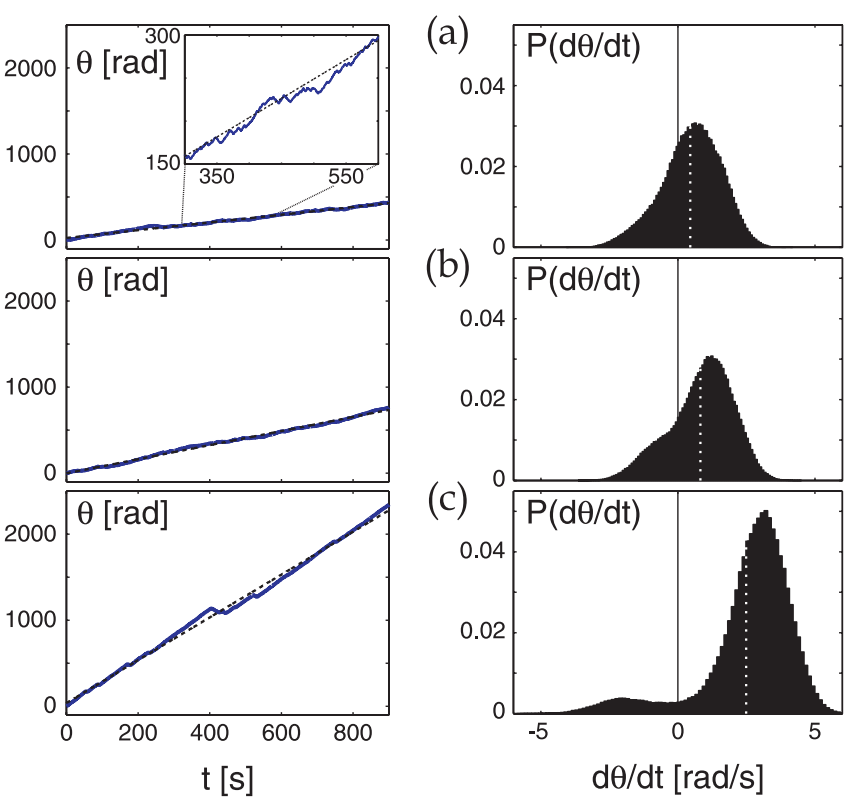

FIG. 3 (color online). Asymmetrically coated vanes: ratchet. The angle $\theta(t)$ and the angular velocity distribution $P(d \theta / d t)$, for $N=2000$ and $h=51 \mathrm{~mm}$ at three different shaking strengths illustrate the ratchet effect: The mean angular velocity $\langle d \theta / d t\rangle$ is indicated by the slope of the straight dashed lines in the $\theta(t)$ plots as well as the vertical white dashed lines in $P(d \theta / d t)$. (a) Amplitude $a=1.5 \mathrm{~mm}$, frequency $f=110 \mathrm{~Hz}$ [S=2.15], the inset shows typical velocity reversals around the average $\langle d \theta / d t\rangle=0.45 \mathrm{rad} / \mathrm{s}$, (b) $a=3.5 \mathrm{~mm}, f=55 \mathrm{~Hz}$ [S=2.92], $\langle d \theta / d t\rangle=0.82 \mathrm{rad} / \mathrm{s}$, (c) $a=3.5 \mathrm{~mm}, f=65 \mathrm{~Hz}$ $[S=4.08],\langle d \theta / d t\rangle=2.49 \mathrm{rad} / \mathrm{s}$. Two movies of the ratchet in action are available as EPAPS_4 and EPAPS_5 [8].

value $S_{\text {cr }}$ to establish the ratchet effect: In Fig. 3(a) the vanes are seen to rotate in the counterclockwise direction with an average angular velocity $\langle d \theta / d t\rangle=0.45 \mathrm{rad} / \mathrm{s}$. The maximum of the velocity distribution lies somewhat higher than this average value, and the inset shows the reason why: Despite its preference for the counterclockwise direction, the system occasionally also moves in the other direction, when due to a fluctuation the collective particle collisions on the four soft sides of the vanes happen to overcome those on the uncoated sides. These reversals are a characteristic feature of the ratchet and remain present also at higher shaking intensities.

In Fig. 3(b) we witness how the velocity distribution has acquired a shoulder, indicating the formation of a double maximum just as in the symmetric case, but now skewed. The fully developed-asymmetric — double peak is seen in Fig. 3(c). The rotating vanes and the induced convection roll in the granular gas reinforce each other, making the motion of the system more persistent, not only when it is rotating in the preferential (ratchet) direction but also when, by a statistical fluctuation, the whole system happens to rotate in the opposite sense. This persistence of the motion also shows up in the $\theta(t)$ plot, which zigzags just as in Fig. 2(d), only now with longer zigs than zags. 
(a)
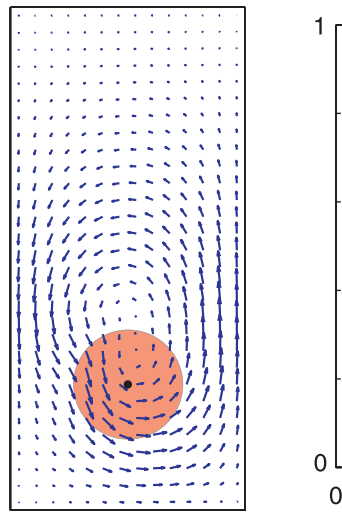

(b)

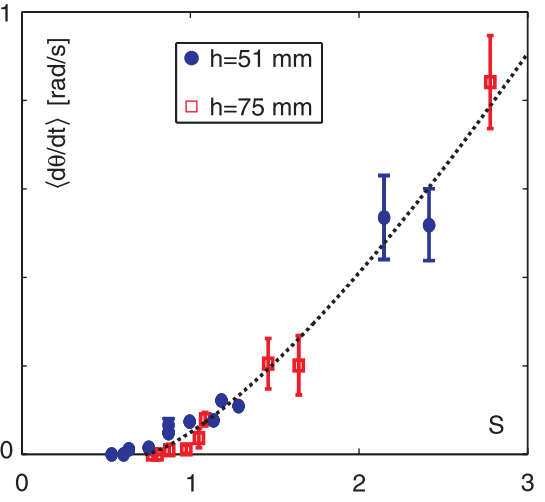

FIG. 4 (color online). (a) Symmetric system (MD simulation). Time-averaged particle flux in the granular gas during a time interval in which the vanes were rotating in the counterclockwise direction. The black dot marks the location of the axis and the shaded area indicates the region in which the vanes rotate. There is a clear convection roll of gas particles rotating in the same (counterclockwise) direction as the vanes. (b) Asymmetrically coated vanes (experiment). Average angular velocity $\langle d \theta / d t\rangle$ vs $S$, showing the onset of the ratchet effect at $S_{\mathrm{cr}}=0.73$. The dots (blue) are experimental data for $h=51 \mathrm{~mm}$, the squares (red) for $h=75 \mathrm{~mm}$, always with $N=2000$ particles. The error bars denote the standard deviation of the three experiments associated with each point. The dotted line is the best fit to the data around the onset, $\langle d \theta / d t\rangle \propto\left(S-S_{\mathrm{cr}}\right)^{\beta}$, with $\beta \approx 1.4$.

The ratchet effect is quantified by the mean angular velocity $\langle d \theta / d t\rangle$, given in Fig. 4(b) as a function of the shaking strength $S$. The plot contains experimental data for two values of the height of the axis above the bottom, $h=$ $51 \mathrm{~mm}$ and $h=75 \mathrm{~mm}$, which are seen to collapse onto a single curve [9].

The onset of the ratchet effect takes place via a continuous phase transition at the critical value $S_{\mathrm{cr}}=0.73 \pm 0.03$. Here the mean speed $\langle d \theta / d t\rangle$ becomes nonzero and starts to grow as

$$
\langle d \theta / d t\rangle \propto\left(S-S_{\mathrm{cr}}\right)^{\beta},
$$

with a critical exponent $\beta \approx 1.4 \pm 0.2$, determined from a best fit to the data around the transition. The fact that this exponent is larger than 1 , meaning that $\langle d \theta / d t\rangle$ starts out from 0 with zero slope, confirms the observed smoothness of the transition. The second transition [the development of a double maximum in the distribution $P(d \theta / d t)$ between $S=2.15$ and 2.92 , connected to the convection roll] within the experimental accuracy does not noticeably influence $\langle d \theta / d t\rangle$, which continues to grow without a clear change of trend.

Conclusion.-We have succeeded in extracting work (a directed motion of the vanes) from the stochastically colliding granular particles. Moreover, unlike related chemical motors on a molecular scale [5], our ratchet is capable of any unlimited number of revolutions. A detailed comparison with the theory presented in [7] will be provided in a forthcoming publication.

An essential new ingredient to make the SmoluchowskiFeynman prototype work has been the use of a granular gas, as it provides a heat bath out of thermal equilibrium. A unique feature of this heat bath is that it is not merely a passive background but in fact interacts actively with the vanes: This is precisely what happens when the system spontaneously develops a convection roll, which proves to be instrumental in sustaining and stabilizing the ratchet motion of the vanes.

We thank Gert-Wim Bruggert, Martin Bos, Jean Walter, and Stijn Klaassen for setting up the experiment and Ralph Eichhorn, Peter Reimann, and Janneke Giele for stimulating discussions. This work is part of the research program of FOM, which is financially supported by NWO.

[1] M. Smoluchowski, Phys. Z. 13, 1069 (1912).

[2] R.P. Feynman, R. B. Leighton, and M. Sands, The Feynman Lectures On Physics (Addison-Wesley, Reading, MA, 1963), Vol. 1, Chap. 46.

[3] P. Reimann, Phys. Rep. 361, 57 (2002); C. Van den Broeck, R. Kawai, and P. Meurs, Phys. Rev. Lett. 93, 090601 (2004); M. van den Broek and C. Van den Broeck, Phys. Rev. E 78, 011102 (2008).

[4] R. D. Astumian and P. Hänggi, Phys. Today 55, 11, 33 (2002).

[5] J. K. Gimzewski et al., Science 281, 531 (1998); T. R. Kelly, J. P. Sestelo, and I. Tellitu, J. Org. Chem. 63, 3655 (1998); N. Koumura et al., Nature (London) 401, 152 (1999); K. L. Sebastian, Phys. Rev. E 61, 937 (2000); R. A. van Delden, Nature (London) 437, 1337 (2005); D. Y. Petrov et al., Biophys. J. 92, 2953 (2007); T. R. Kelly et al., J. Am. Chem. Soc. 129, 376 (2007).

[6] B. Cleuren and C. Van den Broeck, Europhys. Lett. 77, 50003 (2007); G. Costantini, U. M. B. Marconi, and A. Puglisi, Phys. Rev. E 75, 061124 (2007); G. Costantini, U.M. B. Marconi, and A. Puglisi, Europhys. Lett. 82, 50008 (2008); P. Hänggi and F. Marchesoni, Rev. Mod. Phys. 81, 387 (2009).

[7] B. Cleuren and R. Eichhorn, J. Stat. Mech. (2008) P10011.

[8] See supplementary material at http://link.aps.org/ supplemental/10.1103/PhysRevLett.104.248001 for the setup, the molecular dynamics simulation results (including a movie), and three experimental movies of the ratchet.

[9] Because the results for $N=500,1000$ are very similar, in Fig. 4(b) only the results for $N=2000$ have been plotted. 